\title{
Pressure Distribution Analysis of X-Ray Table Mattresses
}

$\underline{\text { Nadi Alresheedi }}{ }^{1}$

Lucy Anne Walton $\underline{1}$

Andrew Tootell ${ }^{1}$

Jo-Anne Webb 1

Peter $\operatorname{Hogg}^{1}$

${ }^{1}$ School of Health and Society, University of Salford, Salford M5 4WT, United Kingdom. 


\begin{abstract}

\section{Background}

There is a risk of developing pressure ulcers from lying on an X-ray table mattress, if the mattress pressure redistribution properties are poor.
\end{abstract}

\title{
Aim:
}

To assess the pressure redistribution properties of 'new' and 'in current clinical use' X-ray table mattresses.

\section{Methods and materials:}

Twenty one X-ray table mattresses, each of $2.5 \mathrm{~cm}$ thickness, were evaluated. An anthropomorphic human phantom of adult stature with five different weights ( minimum, first quartile, mean, third quartile and maximum ) was used to simulate human head, pelvis and heels (pressure ulcer jeopardy areas). Using Xsensor technology, peak pressure was measured and Interface Pressure Ratio was calculated for the three pressure ulcer jeopardy areas 'with' and 'without' an X-ray table mattress.

\section{Results:}

For all mattresses, statistically significant differences $(\mathrm{p}<0.05)$ were found between the peak pressure values with and without using an X-ray table mattress for the three pressure ulcer jeopardy areas; similarly, for all mattresses, statistically significant differences $(p<0.05)$ were found between the Interface Pressure Ratio values with and without using x-ray table mattress. The type and age of the mattress was observed to have an impact on peak pressure values and Interface Pressure Ratios, with older mattresses performing worse.

\section{Conclusion:}

Peak pressure values and Interface Pressure Ratios are reduced significantly when using newer X-ray table mattresses. This could be because newer mattresses use more appropriate materials in their construction and/or older mattresses have lost their pressure redistribution properties. Radiology departments should consider assessing mattresses pressure redistribution properties, perhaps on an annual basis 


\section{Introduction}

Pressure is a significant causative factor in pressure ulcer development [1, 2] as it can impact significantly upon blood flow, which can cause partial or even complete blood vessel occlusion. In turn this can lead to ulcer formation [3]. General bed mattresses, in hospital and community settings, have the potential to lead to the development of pressure ulcers in at risk populations $[4,5,6]$ and considerable research has gone into their design in order to minimise this problem. Similarly, X-ray table mattresses have the potential to lead to the development of pressure ulcers in at risk populations [7], but almost no research has been conducted into the design of these mattresses, with pressure ulcer minimisation in mind.

A predisposing factor to pressure ulcer development is prolonged pressure applied to the skin and underlying structures, 20 minutes is long enough to start the process of tissue breakdown [8]. The time it takes for X-ray imaging procedures varies. A lower limb X-ray would typically take 10 mins, whilst an interventional procedure could require the patient to lie in one position for two hours or more. While the time spent lying on an X-ray table is often short, the thickness of these mattresses (typically being $\sim 2.5 \mathrm{~cm}$ ) is significantly less than trolley and bed mattresses, meaning it could take less time for tissue damage to occur, which could lead to ulcer development or exacerbation of existing ulcers.

No cross-sectional analysis of existing X-ray table mattresses has been published to assess their pressure distribution properties. The current paper applies the methodology of Alresheedi et al [9] to analyse the pressure distribution properties of twenty-one X-ray table mattresses. These mattresses are of varying ages and are currently in use or available for clinical use. Alresheedi's method [9] is novel and uses an adult stature anthropomorphic phantom, with varying weights, together with XSensor interface pressure mapping technology. 


\section{Methods and Materials:}

Four large hospitals in the North West of England and one X-ray table mattress manufacturer were invited to participate in this study. In total, 21 mattresses were included in the study. The manufacturer provided three new X-ray table mattresses, representing their product range. Eighteen mattresses from the four hospitals were included in the study with ages ranging from 1 year to 20 years old. The mean age of the mattresses was 9 years (SD 6.2).

Table 1 displays mattress characteristics in this study. Of the 21 mattresses, 16 were of unknown construction and their manufacturer details were not known. Many mattresses had no identifying information sewn onto them and the departments had no information about them.

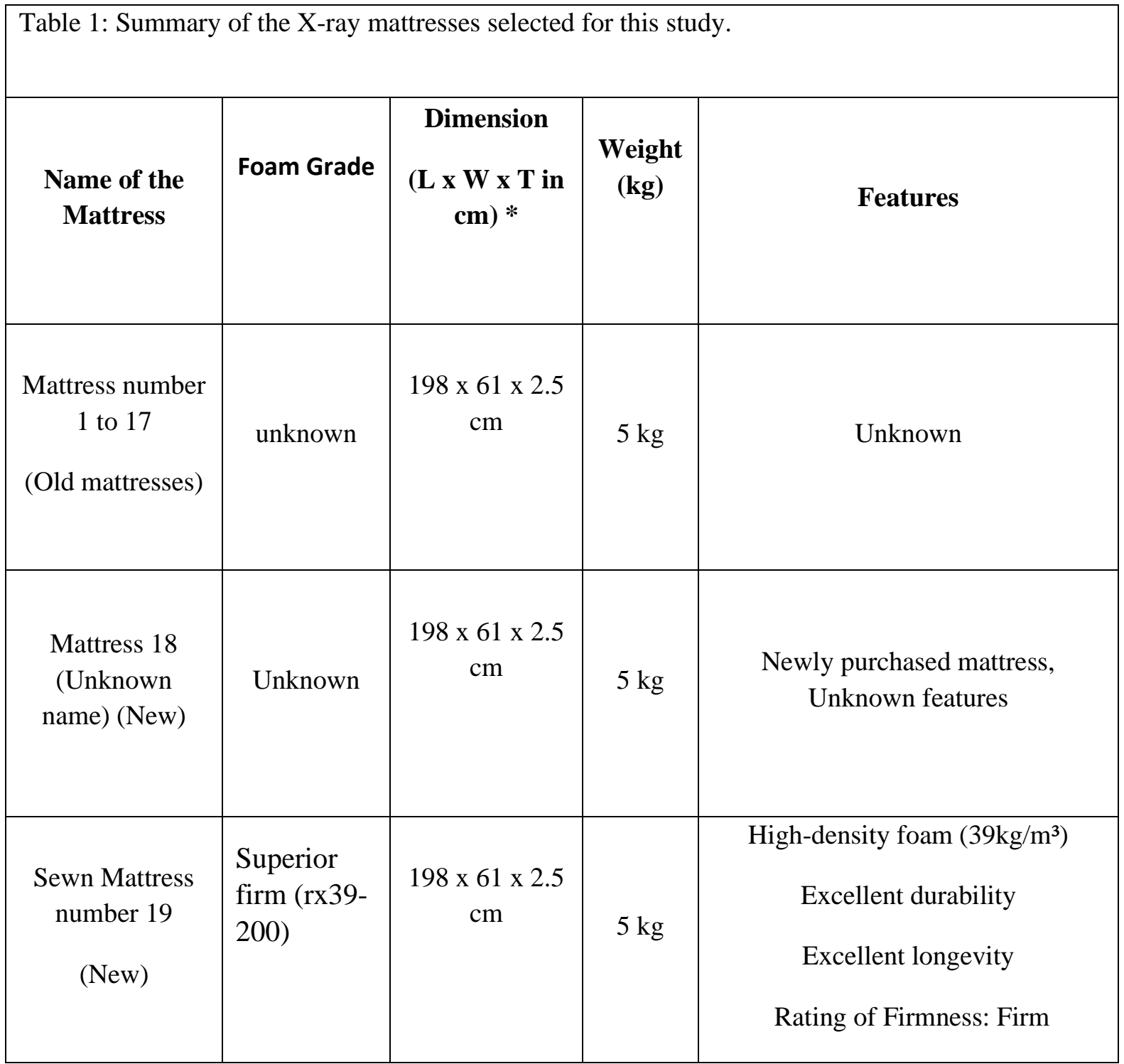




\begin{tabular}{|c|l|c|c|c|}
\hline & & & $\begin{array}{c}\text { Rating of Recovery: Excellent } \\
\text { Premium replacement for fibre } \\
\text { filled cushions }\end{array}$ \\
& & & & $\begin{array}{c}\text { Adheres to strict British fire } \\
\text { regulations [10] }\end{array}$ \\
Anti-static \\
Mattress
\end{tabular}

The pressure analysis method followed that outlined by Alresheedi [9] using a threedimensional (3D) printed phantom with a length of $175 \mathrm{~cm}$. The phantom represented the three main anatomical pressure ulcer jeopardy areas - the head, sacrum and heels (Figure 1). To represent a range of human weights, 5 weights were applied to the phantom and included minimum, first quartile, mean, third quartile and maximum as defined in Alresheedi et al paper [9]. Measurement of Peak Pressure Index (PPI) is a standard metric reported in the literature 
when investigating pressure ulcer risk. For this reason, the PPI was used and measured at the head, sacrum and heels using Xsensor pressure mapping equipment [11] in two conditions with and without an X-ray table mattress. PPI values with and without a mattress were used to calculate Interface Pressure Ratio (IPR). Prior to taking each pressure measurement, a stabilisation period of three minutes was observed whilst phantom, Xsensor and mattress / no mattress were in the experimental condition; this was followed by a 15-minute data collection period. To minimise random error, data collection was repeated three times and then data was averaged, and standard deviations were calculated. IPR was calculated using the following equation.

$$
\mathrm{IPR}=\frac{P P V \text { when } \text { Mattress is used }}{P P V \text { for no mattress }}
$$

IPR varies between 0 and $1(0<\mathrm{IPR} \leq 1)$. An IPR value of 1 indicates the mattress has the same interface pressure redistribution properties as a hard surface (table top); as the IPR approaches 0 , the interface pressure redistribution properties of the mattress improve.

PPI data was analysed using SPSS version 22.0 (IBM Inc, Armonk, New York, US). ShapiroWilk test was used to investigate the normality of the data prior to using any statistical test. The data were found to be normally distributed $(\mathrm{p}<0.05)$ and then Paired T-Test was used to compare PPI with and without a mattress. 


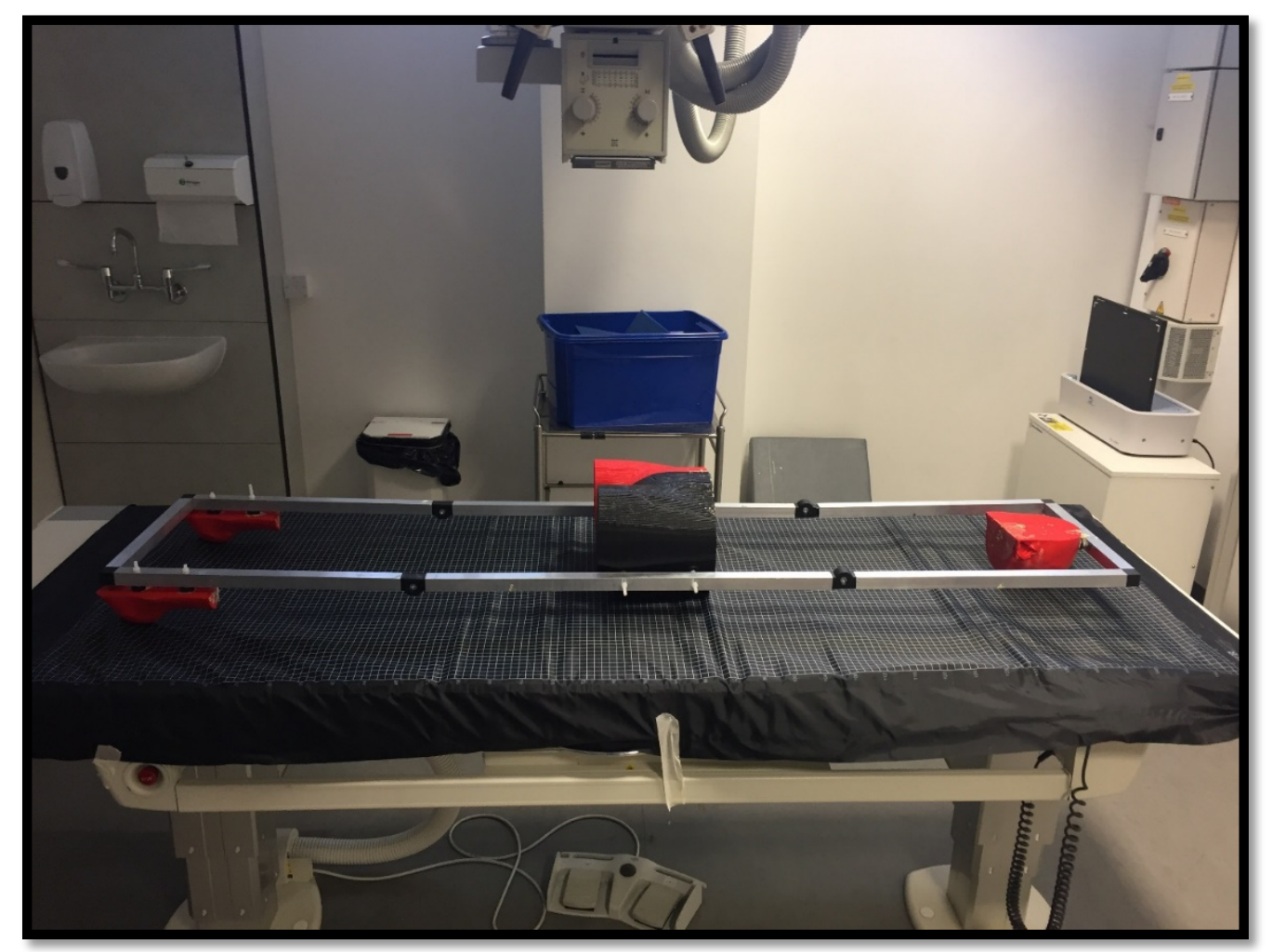

Figure 1: Experimental setup for the X-sensor Px100 system fixed on the X-Ray table with 3D Phantom placed on top.

\section{Results:}

Figures 2-7, and Tables 2-4 (see Appendix) illustrate the variation in PPI and IPR for the head, sacrum, and heels jeopardy areas of the phantom for the five weights. As seen, weight is indicated as minimum, first quartile, mean, third quartile and maximum, in accordance with the method published by [9]. Statistically significant differences $(p<0.05)$ were found between PPI with and without using X-ray table mattress for head, sacrum and heels for all 21 mattresses. The age of the mattress has an impact on PPI, with older mattresses performing worse as indicated in figure 2, 4, and 6 and IPR in figures 3,5, and 7. 


\section{Head}

Figure 2 shows the graphic presentation of PPI of the head phantom for all $2.5 \mathrm{~cm}$ mattresses using the five weight categories. With the exclusion of the hard surface, data is presented with oldest mattress on the left through to newest mattress on the right. The symbols (e.g. '\&') indicate mattress age as \&: 20 years @: 15 years, ¥: 10 years, (c): 8 years, \#: 7 years, £: 6 years, §: 4 years, $\varnothing: 1$ year, А: new.

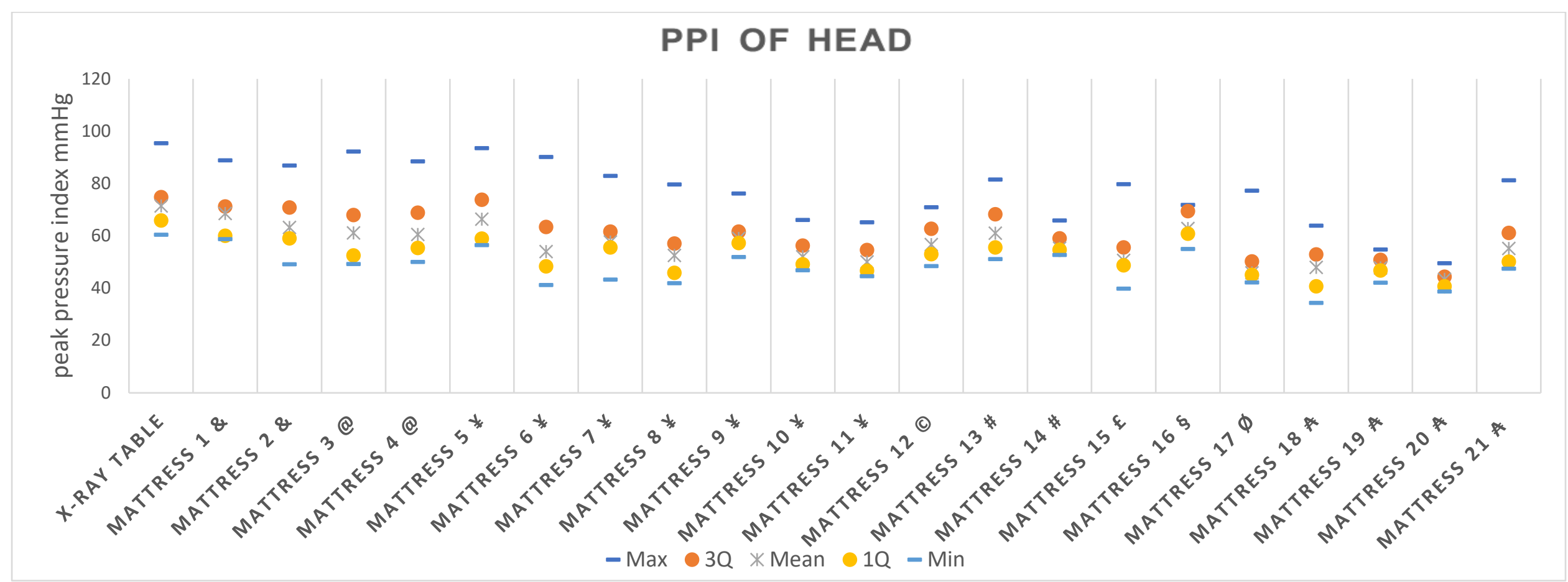

Figure 2: Shows the Peak Pressure index of the 3D phantom Head phantom for the five weight categories. With the exclusion of the hard surface, data is presented with oldest mattress on left through to newest mattress on right. 
The IPR values can be seen in Figure 3, the highest mean value along with $1 \mathrm{Q}$ and 3Q was for Mattress 1 \& the IPR values were 0.96 , 0,91 and 0.95. The lowest IPR for all of them was Mattress 20 with mean and $1 \mathrm{Q}$ and $3 \mathrm{Q}$ value of $0.61,0.62$ and 0.59 .

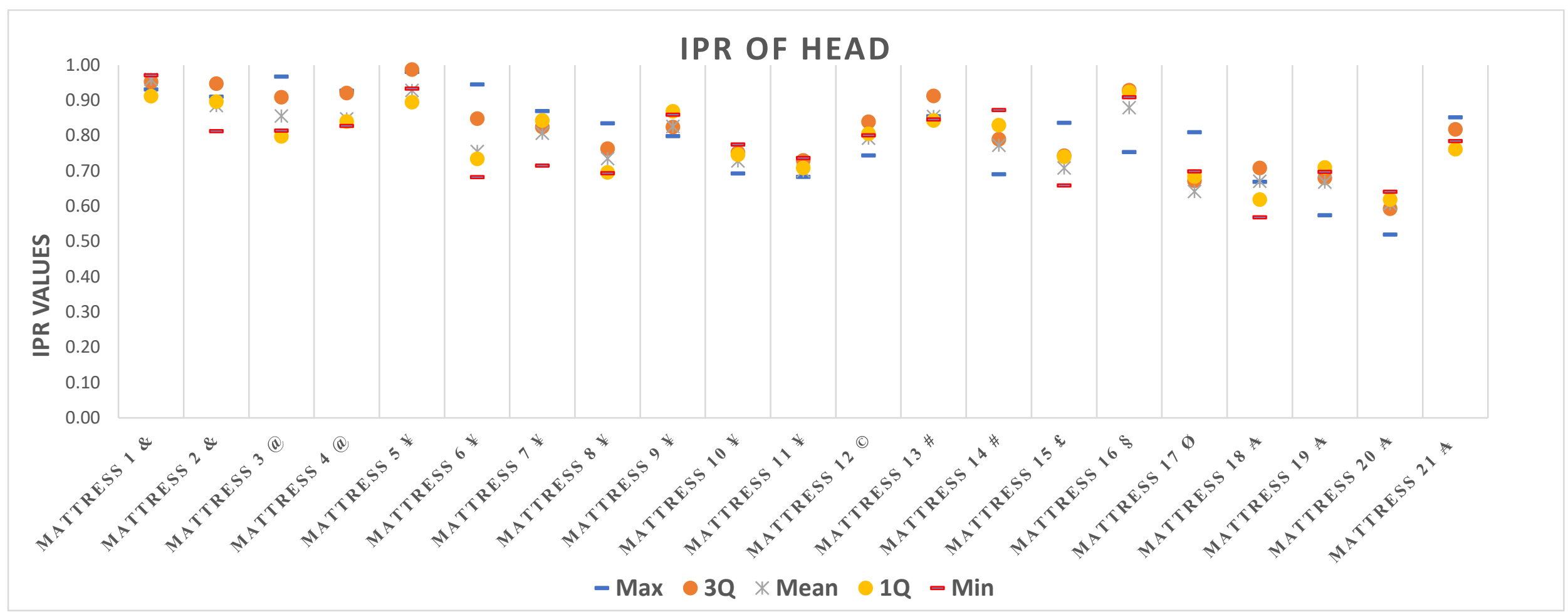

\&: 20 years @: 15 years, ¥: 10 years, ( $: 8$ years, \#: 7 years, $\mathbf{f :} 6$ years, §: 4 years, $\emptyset: 1$ year, $\mathbf{A}:$ new. 


\section{Sacrum}

Figure 4 shows the peak pressure index of the sacrum phantom for all $2.5 \mathrm{~cm}$ mattresses using the five weight categories. With the exclusion of the hard surface, data is presented with oldest mattress on left through to newest mattress on right.

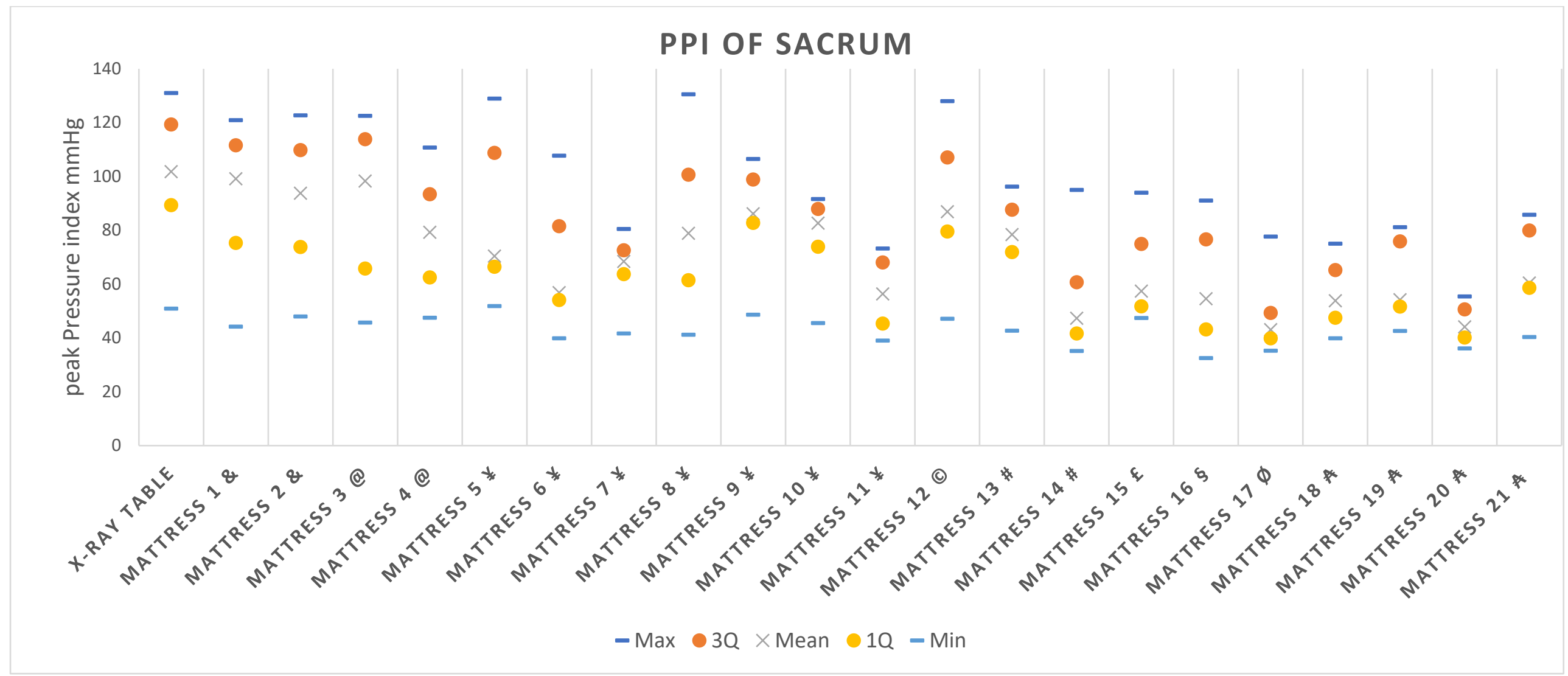

Figure 4: Shows the Peak Pressure Index of the sacrum phantom for the five weight categories. With the exclusion of the hard surface, data is presented with oldest mattress on left through to newest mattress on right. 
Figure 5 showed the mean IPR of Mattress 1 was 0.97 indicating it was the poorest at redistributing pressure and Mattress 20 with the lowest number of weight value with the mean value of 0.43 .The highest mean value along with $1 \mathrm{Q}$ and $3 \mathrm{Q}$ was for Mattress $1 \&$ the IPR values were 0.97 , 0,84 and 0.94. The lowest IPR for all of them was Mattress 20 with mean and $1 \mathrm{Q}$ and $3 \mathrm{Q}$ value of 0.43, 0.45 and 0.42.

\section{Sacrum}

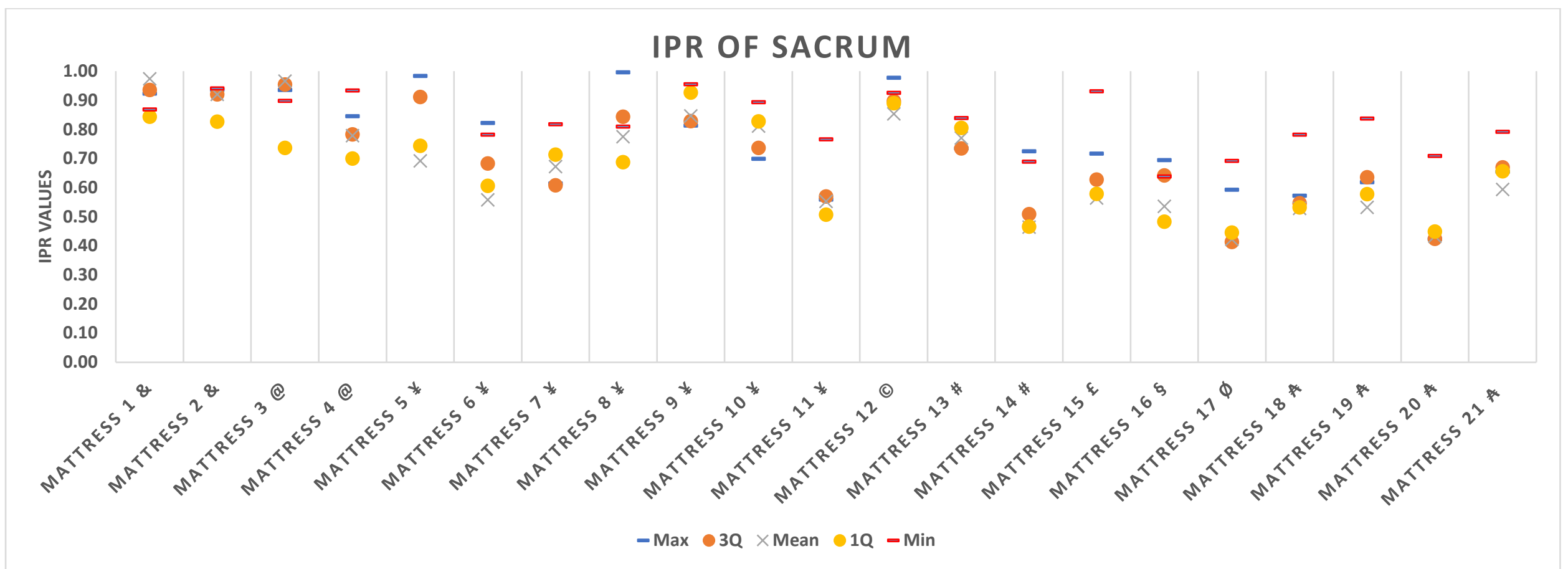

\&: 20 years @: 15 years, ¥: 10 years, @: 8 years, \#: 7 years, $\mathbf{\ddagger}: 6$ years, §: 4 years, $\emptyset: 1$ year, $\mathbf{A}:$ new. 
Heels

In figure 6, the PPI of the Heels phantom for all $2.5 \mathrm{~cm}$ mattresses using the five weight categories is displayed. With the exclusion of the hard surface, data is presented with oldest mattress on left through to newest mattress on right was seen.

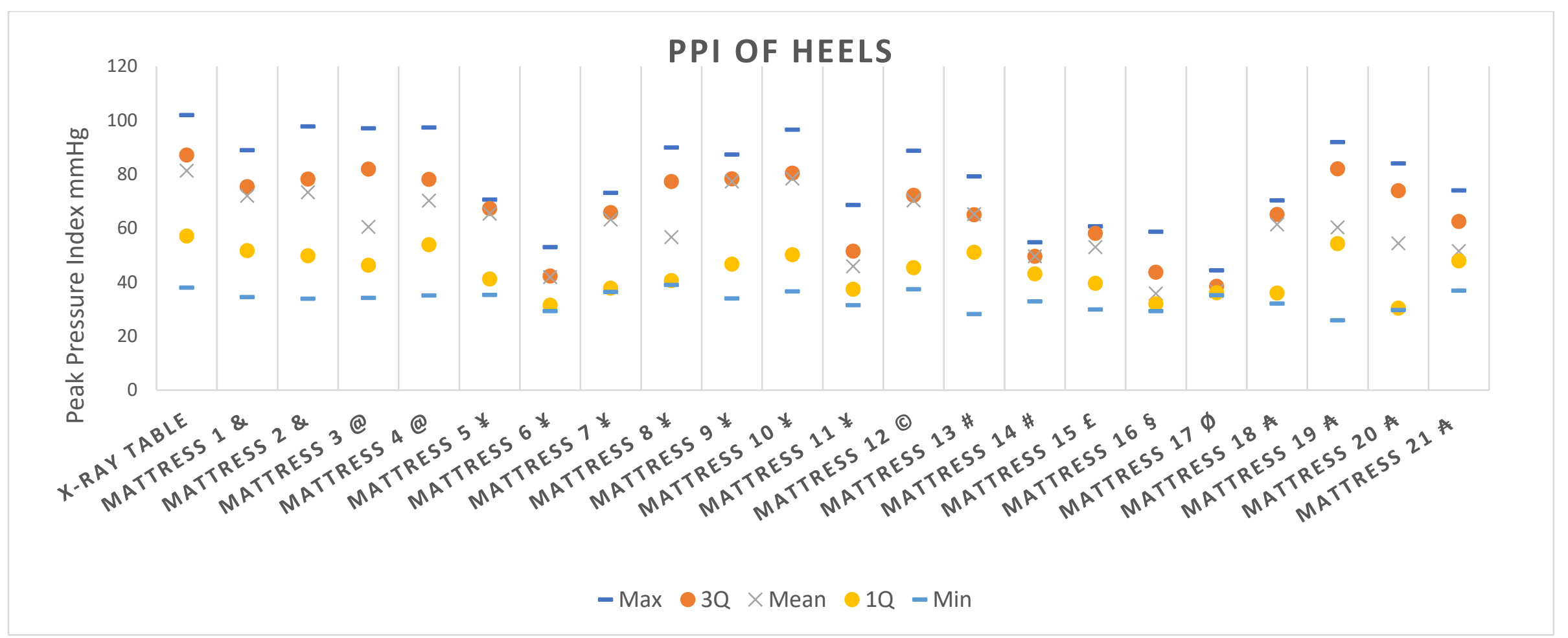

Figure 6: Shows the Peak Pressure Index of the heels phantom for the five weight categories. With the exclusion of the hard surface, data is presented with oldest mattress on left through to newest mattress on right 
Figure 7 shows for the heels, the highest mean weight, 1Q and 3Q value was measured for Mattress 10, the IPR values were $0.96,0.88$ and 0.92.

Whereas, the lowest IPR for the mean weight, $1 \mathrm{Q}$ and $3 \mathrm{Q}$ were $0.52,0.55$ and 0.49 respectively which were found for mattress 6.

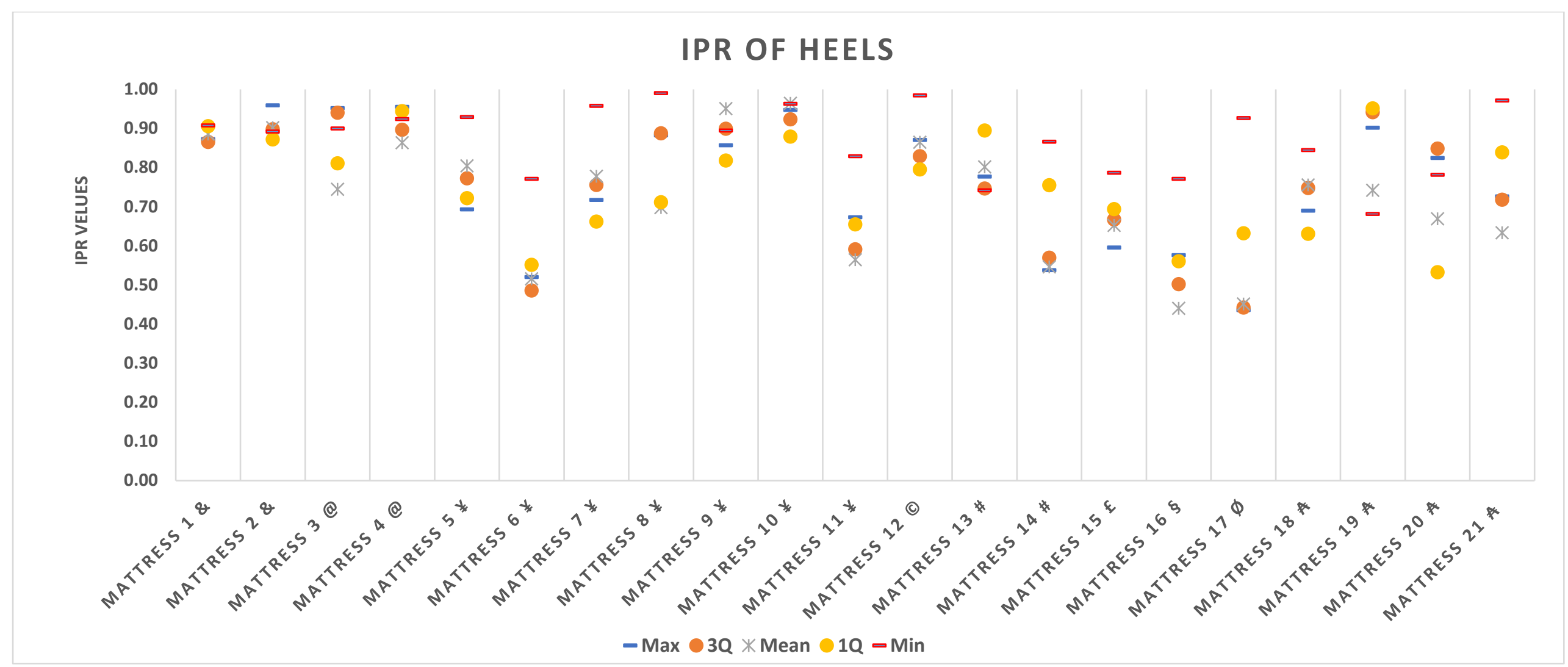

\&: 20 years @: 15 years, ¥: 10 years, @: 8 years, \#: 7 years, $\mathbf{f :} 6$ years, §: 4 years, $\emptyset: 1$ year, $\mathbf{A}:$ new. 


\section{Discussion}

This study focused on assessing new and in-use pressure redistribution properties of $2.5 \mathrm{~cm}$ thick X-ray table mattresses using a phantom. Pressure redistribution capability is taken as a risk indicator for pressure ulcer formation in humans.

Table 2 (Appendix 1) and Figure 2 show PPIs for head, with mean PPI for the hard surface / X-ray table giving the highest values, as expected. The lowest mean PPI is seen in Mattress 20 (A). Table 3 (Appendix 2) and Figure 4 show PPIs for sacrum, highest value is seen for the hard surface. The lowest value was for Mattress 17 Ø. Table 4 (Appendix 3) and Figure 6 show PPIs for heels, highest value was for the hard surface. The lowest value is seen in Mattress 16 $\S$ and Mattress $17 \varnothing$. PPIs vary between mattresses, with newer mattresses generally demonstrating better pressure redistribution properties than older ones. Overall, a wide range of IPR and PPI values exist across the new and in-use $2.5 \mathrm{~cm}$ thick X-ray table mattresses, with newer mattresses tending to have better pressure redistribution properties than older ones see figure 2 and figure 4. Statistically significant differences $(\mathrm{p}>0.05)$ were found for heels, head and sacrum between PPI with and without using an X-ray table mattress.

It has been suggested that the impact of medical imaging and radiotherapy surfaces upon the patients who undergo the radiography/therapy procedures need to be analysed and improved because patients can spend a considerable amount of time on them [12]. The results of this work have importance to radiology departments. For the four hospitals and the new mattresses provided by the manufacturer included in our analysis there is a wide variation in PPI and IPR values, with some performing extremely poorly, for example mattresses aged 15 and 20 years. Extrapolating, we speculate these findings will be similar in other radiology departments and thus there will be many mattresses which will not offer maximum protection to patients who are at risk of developing a pressure ulcer. Given the trend that older mattresses perform worse 
than newer mattresses it raises a question about how long a mattress should be kept in service and whether quality testing of the mattress should be conducted at intervals to ensure their pressure redistribution properties are preserved to a level that is considered adequate for clinical use.

We suggest manufacturers consider using quantitative methods, such as the one outlined in this paper, to inform mattress design and development, with key pressure metrics (e.g. phantom PPI and IPR) being included in the literature which accompanies the product into clinical use. If quality testing for pressure redistribution was introduced as part of the normal clinical routine, then [baseline] key pressure metrics data provided by manufacturers could be used to assess mattress performance over time. Further work needs doing to determine a suitable time interval for performing quality control.

Finally, with pressure redistribution properties in mind, we believe that x-ray table mattresses will have maximum patient weights associated with them. Again, it would be helpful if manufacturers could provide this information. We speculate, particularly because of their thinness, X-ray table mattresses will lose their pressure redistribution priorities beyond certain weights and 'bottom out'. Further research is warranted to identify weight limits for each mattress, and again perhaps this information could be provided by manufacturers.

\section{Conclusion:}

PPI reduces significantly when an X-ray table mattress is used, thus consideration needs giving to determine circumstances when a mattress is not to be used. Given that X-ray mattresses in clinical use display a wide range of pressure distribution properties it is important that clinical standards be established, perhaps using PPI and IPR, for mattress performance and testing to 
occur. Manufacturers should thus provide baseline data, to inform mattress procurement and ongoing quality testing when in clincial use. Using baseline and clinical standards data, decisions can then be made about when to remove mattresses from clinical use.

Future work should focus on examing mattresses of different thicknesses and characteristics, to include those used on trollies, interventional rooms and CT / MR scanners and it is important that clinical standards be established, perhaps using PPI and IPR, for mattress performance and testing to occur. 


\section{Appendix 1}

Table 2 shows the Peak Pressure Index (PPI) for the head phantom. Mean PPI for the hard surface (X-ray table) had the highest values across all human equivalent weights, as expected. The lowest mean PPI was seen for Mattress 20 for the majority of human equivalent weights, which is a new anti-static mattress.

3D phantom - Head:

Table 2: Data shows PPI across the range of [human equivalent] weights for the Head; the symbols (e.g. ' $\&$ ') indicate mattress age.

\begin{tabular}{|c|c|c|c|c|c|}
\hline & \multicolumn{5}{|c|}{ Peak Pressure Index of Head $(\mathrm{mmHg}) \pm S D$} \\
\hline $\begin{array}{l}\text { Mattress name } \\
\text { (thickness } 2.5 \mathrm{~cm} \text { ) }\end{array}$ & $\begin{array}{l}\text { Maximum } \\
\quad \pm S D\end{array}$ & $\begin{array}{c}3^{\text {rd }} \text { quartile } \\
\quad \pm S D\end{array}$ & Mean \pm SD & $\begin{array}{l}1^{\text {st }} \text { quartile } \\
\quad \pm S D\end{array}$ & $\begin{array}{l}\text { Minimum } \\
\quad \pm S D\end{array}$ \\
\hline $\begin{array}{l}\text { Hard Surface / X-ray } \\
\text { table }\end{array}$ & $95.4 \pm 1.8$ & $74.8 \pm 1.2$ & $71.5 \pm 1.5$ & $65.9 \pm 1.5$ & $60.5 \pm 1.8$ \\
\hline Mattress 1 \& & $88.9 \pm 1.4$ & $71.3 \pm 1.5$ & $68.6 \pm 2.4$ & $60.1 \pm 1.8$ & $58.8 \pm 2.6$ \\
\hline Mattress 2 \& & $86.9 \pm 1.9$ & $70.9 \pm 1.1$ & $63.3 \pm 2.9$ & $59.1 \pm 2.4$ & $49.2 \pm 2.0$ \\
\hline Mattress 3 @ & $92.3 \pm 3.6$ & $68 \pm 1$ & $61.2 \pm 1.8$ & $52.6 \pm 2.5$ & $49.3 \pm 1.7$ \\
\hline Mattress $4 @$ & $88.5 \pm 2.5$ & $68.9 \pm 2$ & $60.6 \pm 3.5$ & $55.4 \pm 4$ & $50.1 \pm 3$ \\
\hline Mattress $5 ¥$ & $93.6 \pm 2.4$ & $73.9 \pm 3$ & $66.4 \pm 2.1$ & $59 \pm 1.9$ & $56.5 \pm 2.6$ \\
\hline Mattress $6 ¥$ & $90.2 \pm 2.1$ & $63.5 \pm 1.9$ & $54 \pm 2.1$ & $48.4 \pm 1.7$ & $41.3 \pm 2.7$ \\
\hline Mattress $7 ¥$ & $83 \pm 0.7$ & $61.7 \pm 1.2$ & $57.7 \pm 2.2$ & $55.6 \pm 1.5$ & $43.3 \pm 2.1$ \\
\hline Mattress $8 ¥$ & $79.7 \pm 3.5$ & $57.1 \pm 2$ & $52.6 \pm 1.5$ & $45.9 \pm 1$ & $42 \pm 2.2$ \\
\hline Mattress $9 ¥$ & $76.2 \pm 1.5$ & $61.7 \pm 1.7$ & $59.1 \pm 1.9$ & $57.3 \pm 2.2$ & $52 \pm 1.6$ \\
\hline Mattress $10 ¥$ & $66.1 \pm 2.6$ & $56.3 \pm 3.2$ & $52.1 \pm 1.5$ & $49.2 \pm 2.5$ & $46.9 \pm 2$ \\
\hline Mattress $11 ¥$ & $65.2 \pm 1.2$ & $54.6 \pm 1.9$ & $50.1 \pm 1.3$ & $46.7 \pm 1.9$ & $44.6 \pm 1.4$ \\
\hline Mattress 12 C & $71 \pm 1.4$ & $62.8 \pm 1.2$ & $56.7 \pm 2.1$ & $53.1 \pm 1.1$ & $48.5 \pm 1.6$ \\
\hline
\end{tabular}




\begin{tabular}{|l|l|l|l|r|r|}
\hline Mattress 13 \# & $81.6 \pm 1.5$ & $68.3 \pm 1.3$ & $61.1 \pm 1$ & $55.6 \pm 2.3$ & $51.2 \pm 2.3$ \\
\hline Mattress 14 \# & $65.9 \pm 1.4$ & $59.1 \pm 1.8$ & $55.3 \pm 1.1$ & $54.7 \pm 1.1$ & $52.8 \pm 2.4$ \\
\hline Mattress 15 § & $79.8 \pm 1.7$ & $55.6 \pm 2.2$ & $50.7 \pm 1.6$ & $48.8 \pm 2.1$ & $39.9 \pm 1.2$ \\
\hline Mattress 16 § & $71.9 \pm 2.7$ & $69.5 \pm 1.6$ & $62.9 \pm 1.4$ & $60.9 \pm 1.5$ & $55 \pm 2$ \\
\hline Mattress 17 Ø & $77.3 \pm 2.4$ & $50.3 \pm 1.7$ & $45.9 \pm 2.1$ & $45.1 \pm 1.8$ & $42.3 \pm 1.6$ \\
\hline Mattress 18 A & $63.9 \pm 1.4$ & $53 \pm 1.6$ & $48 \pm 2.1$ & $40.8 \pm 1.8$ & $34.4 \pm 1.7$ \\
\hline Mattress 19 A & $54.8 \pm 2.1$ & $50.9 \pm 2.3$ & $47.8 \pm 1.5$ & $46.8 \pm 1.5$ & $42.2 \pm 1$ \\
\hline Mattress 20 A & $49.6 \pm 1.1$ & $44.4 \pm 1.1$ & $43.4 \pm 0.7$ & $40.8 \pm 0.9$ & $38.8 \pm 1.4$ \\
\hline Mattress 21 A & $81.3 \pm 1.6$ & $61.2 \pm 2.6$ & $55.2 \pm 1.4$ & $50.2 \pm 1.1$ & $47.5 \pm 1$ \\
\hline
\end{tabular}

\&: 20 years @: 15 years, ¥: 10 years, @: 8 years, \#: 7 years, $\mathbf{f : ~} 6$ years, §: 4 years, $\emptyset: 1$ year, А: new. 


\section{Appendix 2}

In Table 3, the data shows PPI across the range of human equivalent weights for phantom sacrum for all $2.5 \mathrm{~cm}$ thick X-ray table mattresses. The highest mean PPI value can be seen for the hard surface. The lowest value for the mean weight was seen for Mattress 17.

3D phantom - Sacrum:

Table 3: Data shows PPI across the range of [sand] weights for 3D phantom Sacrum; the symbols (e.g. ' $\&$ ') indicate mattress age.

\begin{tabular}{|c|c|c|c|c|c|}
\hline & Peak Pressure & dex of Sacru & $(\mathrm{mmHg}) \pm \mathrm{S}$ & & \\
\hline $\begin{array}{l}\text { Mattress name (thickness } \\
2.5 \mathrm{~cm} \text { ) }\end{array}$ & Maximum $\pm S D$ & $\begin{array}{c}3^{\text {rd }} \text { quartile } \\
\quad \pm S D\end{array}$ & Mean \pm SD & $\begin{array}{l}1^{\text {st }} \text { quartile } \\
\pm S D\end{array}$ & $\begin{array}{l}\text { Minimum } \\
\pm S D\end{array}$ \\
\hline Hard Surface / X-ray table & $131 \pm 2.4$ & $119.3 \pm 1.8$ & $101.8 \pm 1.4$ & $89.3 \pm 1.6$ & $50.9 \pm 0.8$ \\
\hline Mattress 1 \& & $120.9 \pm 3.6$ & $111.6 \pm 3.5$ & $99.1 \pm 1.5$ & $75.3 \pm 1.9$ & $44.2 \pm 1.7$ \\
\hline Mattress 2 \& & $122.7 \pm 4.9$ & $109.8 \pm 3.6$ & $93.7 \pm 2.6$ & $73.8 \pm 2.6$ & $47.9 \pm 1.2$ \\
\hline Mattress 3 @ & $122.5 \pm 3.0$ & $113.8 \pm 0.9$ & $98.3 \pm 1.2$ & $65.8 \pm 1.4$ & $45.7 \pm 2.9$ \\
\hline Mattress $4 @$ & $110.7 \pm 3.6$ & $93.4 \pm 1.4$ & $79.2 \pm 2.6$ & $62.5 \pm 2.4$ & $47.5 \pm 3.1$ \\
\hline Mattress $5 ¥$ & $128.9 \pm 4.8$ & $108.7 \pm 1.9$ & $70.4 \pm 1.9$ & $66.4 \pm 2.8$ & $51.8 \pm 2.2$ \\
\hline Mattress $6 ¥$ & $107.7 \pm 1.8$ & $81.5 \pm 1.7$ & $56.8 \pm 1.8$ & $54.1 \pm 4.2$ & $39.8 \pm 2.7$ \\
\hline Mattress $7 ¥$ & $80.5 \pm 1.5$ & $72.5 \pm 0.9$ & $68.4 \pm 1.7$ & $63.7 \pm 1.7$ & $41.6 \pm 1.6$ \\
\hline Mattress $8 ¥$ & $130.5 \pm 3.9$ & $100.6 \pm 2.7$ & $78.9 \pm 2.6$ & $61.4 \pm 1.8$ & $41.2 \pm 2.1$ \\
\hline Mattress $9 ¥$ & $106.5 \pm 2.9$ & $98.8 \pm 2.8$ & $86.1 \pm 2.1$ & $82.7 \pm 0.7$ & $48.6 \pm 0.9$ \\
\hline Mattress $10 ¥$ & $91.6 \pm 2.9$ & $87.9 \pm 2.5$ & $82.6 \pm 1.3$ & $73.9 \pm 2.1$ & $45.5 \pm 2.3$ \\
\hline Mattress $11 ¥$ & $73.2 \pm 1.9$ & $68 \pm 1.2$ & $56.3 \pm 1.2$ & $45.3 \pm 2.4$ & $39 \pm 1.2$ \\
\hline Mattress 12 ○ & $128 \pm 2.4$ & $107 \pm 1.3$ & $86.9 \pm 2.6$ & $79.5 \pm 1.3$ & $47.1 \pm 1.4$ \\
\hline Mattress 13 \# & $96.2 \pm 1.5$ & $87.6 \pm 1.8$ & $78.4 \pm 1.6$ & $71.9 \pm 1.4$ & $42.7 \pm 1.2$ \\
\hline Mattress 14 \# & $95 \pm 1.8$ & $60.7 \pm 1.3$ & $47.3 \pm 1.3$ & $41.6 \pm 2.2$ & $35.1 \pm 1.0$ \\
\hline Mattress $15 f$ & $93.9 \pm 3.0$ & $74.9 \pm 2.4$ & $57.4 \pm 1.4$ & $51.7 \pm 0.8$ & $47.4 \pm 3.2$ \\
\hline Mattress $16 \S$ & $91 \pm 1.5$ & $76.6 \pm 0.8$ & $54.5 \pm 1.1$ & $43.1 \pm 1.1$ & $32.5 \pm 2.0$ \\
\hline
\end{tabular}




\begin{tabular}{|l|c|c|c|c|c|}
\hline Mattress 17 $\varnothing$ & $77.6 \pm 2.9$ & $49.3 \pm 1.3$ & $43 \pm 1.3$ & $39.8 \pm 1.2$ & $35.2 \pm 1.3$ \\
\hline Mattress 18 A & $75 \pm 1.4$ & $65.2 \pm 1.7$ & $53.8 \pm 1.7$ & $47.5 \pm 1.8$ & $39.8 \pm 1.2$ \\
\hline Mattress 19 A & $81.1 \pm 2.1$ & $75.8 \pm 1.3$ & $54.2 \pm 1.3$ & $51.6 \pm 1.9$ & $42.6 \pm 2.4$ \\
\hline Mattress 20 A & $55.4 \pm 1.8$ & $50.6 \pm 1.4$ & $44.1 \pm 1.5$ & $40.1 \pm 1.5$ & $36.1 \pm 1.0$ \\
\hline Mattress 21 A & $85.7 \pm 1.9$ & $79.9 \pm 1.5$ & $60.4 \pm 1.7$ & $58.6 \pm 1.8$ & $40.3 \pm 1.7$ \\
\hline
\end{tabular}

\&: 20 years @: 15 years, ¥: 10 years, @: 8 years, \#: 7 years, $\mathbf{f :} 6$ years, §: 4 years, $\emptyset: 1$ year, А: new. 


\section{Appendix 3}

In Table 4, the data shows PPI across the range of human equivalent weights for the heels for all $2.5 \mathrm{~cm}$ thick X-ray table mattresses. The highest mean equivalent was for the hard surface. The lowest value seen for the mean equivalent weight was for mattress 16 .

3D phantom - Heels:

Table 4: Data shows PPI across the range of [sand] weights for 3D phantom Heels; the symbols (e.g. ' \&') indicate mattress age

\begin{tabular}{|c|c|c|c|c|c|}
\hline & \multicolumn{5}{|c|}{ Peak Pressure Index of Heels $(\mathrm{mmHg}) \pm S D$} \\
\hline $\begin{array}{l}\text { Mattress name (thickness } 2.5 \\
\mathrm{~cm} \text { ) }\end{array}$ & Maximum $\pm S D$ & $\begin{array}{c}3^{\text {rd }} \text { quartile } \\
\pm S D\end{array}$ & Mean \pm SD & $\begin{array}{c}1^{\text {st }} \text { quartile } \\
\pm S D\end{array}$ & $\begin{array}{l}\text { Minimum } \\
\pm \text { SD }\end{array}$ \\
\hline Hard Surface / X-ray table & $101.9 \pm 1.9$ & $87.1 \pm 0.9$ & $81.3 \pm 2.5$ & $57.1 \pm 1.1$ & $38 \pm 1.2$ \\
\hline Mattress 1 \& & $88.9 \pm 1.0$ & $75.4 \pm 1.6$ & $72 \pm 2.0$ & $51.7 \pm 1.9$ & $34.5 \pm 2.3$ \\
\hline Mattress 2 \& & $97.7 \pm 2.4$ & $78.2 \pm 1.9$ & $73.3 \pm 1.8$ & $49.8 \pm 1.5$ & $33.9 \pm 1.3$ \\
\hline Mattress $3 @$ & $97 \pm 2.4$ & $81.9 \pm 0.5$ & $60.5 \pm 1.7$ & $46.3 \pm 1.9$ & $34.2 \pm 1.1$ \\
\hline Mattress 4 @ & $97.3 \pm 3.5$ & $78.1 \pm 2.3$ & $70.2 \pm 2.4$ & $53.9 \pm 3.5$ & $35.1 \pm 2.4$ \\
\hline Mattress $5 ¥$ & $70.6 \pm 2.2$ & $67.3 \pm 2.4$ & $65.4 \pm 2.0$ & $41.2 \pm 2.5$ & $35.3 \pm 1.8$ \\
\hline Mattress $6 ¥$ & $53 \pm 1.7$ & $42.3 \pm 2.1$ & $41.9 \pm 1.3$ & $31.5 \pm 3.1$ & $29.3 \pm 1.8$ \\
\hline Mattress $7 ¥$ & $73.1 \pm 2.0$ & $65.8 \pm 2.8$ & $63.2 \pm 2.7$ & $37.8 \pm 2.3$ & $36.4 \pm 1.2$ \\
\hline Mattress $8 ¥$ & $89.9 \pm 4.1$ & $77.3 \pm 2.6$ & $56.7 \pm 1.7$ & $40.6 \pm 2.5$ & $39 \pm 2.1$ \\
\hline Mattress $9 ¥$ & $87.3 \pm 0.8$ & $78.3 \pm 1.8$ & $77.3 \pm 1.4$ & $46.7 \pm 1.2$ & $34 \pm 1.9$ \\
\hline Mattress $10 ¥$ & $96.5 \pm 0.9$ & $80.4 \pm 2.0$ & $78.4 \pm 1.3$ & $50.2 \pm 1.3$ & $36.6 \pm 2.1$ \\
\hline Mattress $11 ¥$ & $68.6 \pm 1.7$ & $51.5 \pm 1.9$ & $45.9 \pm 1.2$ & $37.4 \pm 1.8$ & $31.5 \pm 1.7$ \\
\hline Mattress 12 @ & $88.7 \pm 1.9$ & $72.2 \pm 1.5$ & $70.3 \pm 1.3$ & $45.4 \pm 2.9$ & $37.4 \pm 1.6$ \\
\hline Mattress 13 \# & $79.2 \pm 1.8$ & $65 \pm 1.7$ & $65.2 \pm 1.7$ & $51.1 \pm 1.5$ & $28.2 \pm 1.5$ \\
\hline Mattress 14 \# & $54.8 \pm 1.4$ & $49.6 \pm 1.6$ & $44.4 \pm 2.1$ & $43.1 \pm 1.2$ & $32.9 \pm 2.3$ \\
\hline Mattress $15 \mathrm{f}$ & $60.7 \pm 0.9$ & $58.1 \pm 1.9$ & $53 \pm 1.6$ & $39.6 \pm 2.2$ & $29.9 \pm 1.9$ \\
\hline
\end{tabular}




\begin{tabular}{|l|l|l|l|l|l|}
\hline Mattress 16 § & $58.7 \pm 1.2$ & $43.7 \pm 1.5$ & $35.8 \pm 2.1$ & $32 \pm 1.5$ & $29.3 \pm 1.3$ \\
\hline Mattress 17 $\varnothing$ & $44.4 \pm 2.0$ & $38.5 \pm 0.6$ & $36.7 \pm 1.2$ & $36.1 \pm 1.7$ & $35.2 \pm 1.6$ \\
\hline Mattress 18 A & $70.3 \pm 0.6$ & $65.1 \pm 1.3$ & $61.4 \pm 1.5$ & $36 \pm 1.4$ & $32.1 \pm 1.5$ \\
\hline Mattress 19 A & $91.9 \pm 2.6$ & $82 \pm 0.9$ & $60.3 \pm 1.7$ & $54.3 \pm 2.6$ & $25.9 \pm 2.3$ \\
\hline Mattress 20 A & $84 \pm 1.2$ & $73.9 \pm 1.3$ & $54.4 \pm 1.8$ & $30.4 \pm 1.1$ & $29.7 \pm 1.0$ \\
\hline Mattress 21 A & $76.2 \pm 1.2$ & $46 \pm 2.6$ & $41.4 \pm 1.4$ & $39.8 \pm 1.0$ & $33.4 \pm 1.3$ \\
\hline
\end{tabular}

\&: 20 years @: 15 years, ¥: 10 years, @: 8 years, \#: 7 years, $\mathbf{f : ~} 6$ years, §: 4 years, $\emptyset: 1$ year, A: new. 
References

1. AGRAWAL, K. \& CHAUHAN, N. 2012. Pressure ulcers: Back to the basics. Indian Journal of Plastic Surgery, 45, 244-254.

2. MESSER, M. S. 2012. Development of a Tool for Pressure Ulcer Risk Assessment and Preventive Interventions in Ancillary Services Patients. Doctor of Philosophy, University of South Florida.

3. DEMARRE, L., VANDERWEE, K., DEFLOOR, T., VERHAEGHE, S., SCHOONHOVEN, L. \& BEECKMAN, D. 2012. Pressure ulcers: knowledge and attitude of nurses and nursing assistants in Belgian nursing homes. Journal of Clinical Nursing, 21, 1425-1434.

4. Angmorterh SK, England A, Webb J, Szczepura K, Stephens M, Anaman-Torgbor J, Ofori EK, Hogg P. An Investigation of Pressure Ulcer Risk, Comfort, and Pain in Medical Imaging. J Med Imaging Radiat Sci. 2019 Mar;50(1):43-52. doi: 10.1016/j.jmir.2018.07.003. Epub 2018 Aug 9. PMID: 30777247.

5. Bordier C, Klausz R, Desponds L. Patient dose map indications on interventional X-ray systems andvalidation with gafchromic XR-RV3 film. Radiat Prot Dosimetry. 2014;

6. Tugwell JR, England A, Hogg P. Antero-posterior (AP) pelvis x-ray imaging on a trolley: Impact of trolley design, mattress design and radiographer practice on image quality and radiation dose. Radiography (Lond). 2017 Aug;23(3):242-248. doi: 10.1016/j.radi.2017.04.002. Epub 2017 Apr 28. PMID: 28687293.

7. Angmorterh, S.K., 2016. An investigation into interface pressure (IP) risk of healthy volunteers on modern medical imaging and radiotherapy tables (Doctoral dissertation, University of Salford).

8. Moore ZE, Cowman S. Risk assessment tools for the prevention of pressure ulcers. Cochrane Database Syst Rev. 2014 Feb 5;(2):CD006471. doi: 10.1002/14651858.CD006471.pub3. Update in: Cochrane Database Syst Rev. 2019 Jan 31;1:CD006471. PMID: 24497383. 
9. Alresheedi, N., Walton, A.L., Tootell, A.K., Webb, J. and Hogg, P., 2020. A phantombased method to assess X-ray table mattress interface pressures. Journal of Medical Imaging and Radiation Sciences

10. Foamrite. 2020. Buy Foam And Rubber Sheets And Products Online / Foamrite. [online] Available at: <https://www.foamrite.co.uk/> [Accessed 26 June 2020].

11. Sumed International, UK. 2014. Products and specifications. Suzuki, K., and Yamashita,

12. AHMED, A. N., DANSEREAU, J., EAKIN, P., HAASTERT, B. \& PADULA, W. V. 2012. Equipment performance and radiation protection status in X-ray fluoroscopy units in Sudan. Radiation Protection Dosimetry, 148, 174-180. 